\title{
From hierarchies to well-foundedness
}

\author{
Dandolo Flumini · Kentaro Sato
}

Received: date / Accepted: date

\begin{abstract}
We highlight that the connection of well-foundedness and recursive definitions is more than just convenience. While the consequences of making well-foundedness a sufficient condition for the existence of hierarchies (of various complexity) have been extensively studied, we point out that (if parameters are allowed) well-foundedness is a necessary condition for the existence of hierarchies e.g. that even in an intuitionistic setting $\left(\Pi_{1}^{0}-\mathrm{CA}_{0}\right)_{\alpha} \vdash \mathrm{wf}(\alpha)$ where $\left(\Pi_{1}^{0}-\mathrm{CA}_{0}\right)_{\alpha}$ stands for the iteration of $\Pi_{1}^{0}$ comprehension (with parameters) along some ordinal $\alpha$ and $\operatorname{wf}(\alpha)$ stands for the well-foundedness of $\alpha$.
\end{abstract}

Keywords Transfinite Recursion · Well-foundedness · Second Order Arithmetic · Second Order Set Theory · Pseudohierarchy · Intuitionistic Logic

Mathematics Subject Classification (2000) 03B30 - 03D75 - 03E70 • $03 \mathrm{~F} 35$

\section{Introduction}

Theories involving transfinite iterations of comprehension schemata have a long tradition in proof theory, reverse mathematics and generally in foundations of mathematics (e.g. [8, §I.11] and [1, §6 particularly after (6.18)]).

The transfinite recursion theorem usually guarantees that for any wellfounded relation $\prec$ and any $G$ from a given family of operators, there is a

Dandolo Flumini

Zurich University of Applied Sciences, School of Engineering

Technikumstrasse 9, 8400 Winterthur, Switzerland

E-mail: dandoloflumini@gmail.com

Kentaro Sato

University of Bern, Institut für Informatik und angewandte Mathematik

Neubrückstrasse 10, 3012 Bern, Switzerland

E-mail: sato@iam.unibe.ch 
function $F$ such that for all $x$ in the field of $\prec$

$$
F(x)=G(F\lceil\{y \mid y \prec x\}) .
$$

Commonly, right after introducing the theorem, it is proved by induction that $F$ as described above is (if exists) uniquely determined by $\prec$ and $G$. Thus the theorem is often referred to as defining $F$ recursively by (iterating) $G$ along $\prec$. We will see, under very modest assumptions, that the well-foundedness of $\prec$ is a necessary condition for recursive definitions along $\prec$.

The research program "Subsystems of Second Order Arithmetic and Set Theory" (cf. [4]) from which this paper emerged, merely as a byproduct, can be briefly outlined by quoting the leading question as stated in [4]:

What happens if we replace Peano arithmetic and subsystems of second order arithmetic by Zermelo-Fraenkel set theory (with or without the axiom of choice) and subsystems of Morse-Kelley theory of sets and classes, respectively? Which proof-theoretic results have direct analogues and for which results do such analogues not exist?

As it turns out, well orders have applications in arithmetic that have no direct analogs in theories of sets and classes mainly due to the fact that, unlike in arithmetic, being well-founded is not a $\Pi_{1}^{1}$ complete predicate in the set theoretic context (see Remark 6). As a consequence, finding appropriate set theoretic counterparts of theories which heavily rely on the notion of wellfoundedness (such as ATR ${ }_{0}$ ), becomes intricate matter (at least if it is desired that their strength relative to other theories resembles the situation in arithmetic). In early attempts to find the right set theoretic analog of the theory $\mathrm{ATR}_{0}$, the authors experimented with principles that allowed iterations along ill-founded relations. Ultimately, as presented here (see Theorem 1), the studies led to the observation that it is impossible to generalize principles of recursion by extending their domain beyond the well-founded (see also Subsection 4.2). ${ }^{1}$

Our theorem is proved by deriving the well-foundedness of the relation along which the existence of hierarchies is assumed. In particular, if we apply our reasoning to the theory $\left(\Pi_{1}^{0}-\mathrm{CA}\right)_{\alpha}$, we can conclude $\left(\Pi_{1}^{0}-\mathrm{CA}\right)_{\alpha} \vdash \mathrm{wf}(\alpha)$. Since our proof is carried out in pure minimal second order logic ${ }^{2}$, the reasoning also applies to the intuitionistic setting. In spite of the simplicity of our argument, apparently the result presented here was not previously known. This seems evident from the fact that in the literature theories that claim transfinite recursion along some given relation, redundantly, also claim that the relation be well-founded (cf. [5, preliminary definitions in Subsection 2.4 $]^{3}$ ).

At very first sight, the result seems to contradict the $\Pi_{1}^{1}$ completeness of

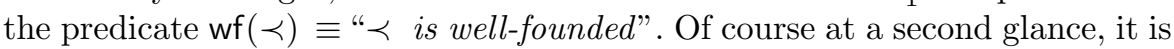

\footnotetext{
1 Different approaches in generalizing recursion principles are effective (see e.g. [7]).

2 By second order logic we mean two-sorted first order logic. Particularly, when we say that our proof is carried out in pure second order logic, then we only use first order axioms.

3 Montalbán, one of the authors of [5], however pointed out to the authors of the present paper, that in the case of classical arithmetic, our result follows from the relativized version of the main theorem in [9]. This connection will be discussed in Subection 4.5.
} 
evident that there is no contradiction. More explicitly, consider the statement

$$
\Phi(\prec) \equiv \exists F \forall x(F(x)=G(F\lceil\{y \mid y \prec x\}))
$$

where $G(X)=\{x \mid \varphi(X, x)\}$ for some fixed elementary formula $\varphi(X, x)$. In this situation, $\Phi(\prec) \longleftrightarrow \mathrm{wf}(\prec)$ contradicts the $\Pi_{1}^{1}$ completeness of wf. However, we have to allow the formula $\varphi(Y, X, x)$ to have an additional free second order variable $Y$, and need the universal closure $\forall Y \Phi(Y, \prec)$ to derive $\operatorname{wf}(\prec)$. The contradiction is thus resolved (cf. Subsection 4.2). In particular, our proof does not work in the parameter-free setting. ${ }^{4}$ However, the type of necessary parameters varies, depending on the contexts, as discussed in Subsection 4.4.

\section{Preliminaries}

Definition 1 We work in the language of second order logic which is an instance of two sorted first order logic. Besides the usual logical symbols (including equality for the first order), we use two types of variables, lower case letters for what we call first order variables and upper case letters that we will refer to as second order variables. The atomic formulas are either of the form $x=y$ where $x, y$ are first order variables, or of the form $X\left(y_{1}, \ldots, y_{n}\right)$ where $X$ is an $n$-ary second order variable and $y_{1}, \ldots, y_{n}$ are first order variables. More complex formulas are build up as usual by means of logical connectives and quantifications. Our base system is minimal logic, i.e. the axioms of first order intuitionistic logic minus the $\perp$-axiom.

Remark 1 Our base theory is "pure" minimal logic without any mathematical (non-logical) axioms. No comprehension axiom is counted as a logical axiom.

While every second order variable has a fixed arity, we will relinquish to denote explicitly arity in a variable because it will always be clear from the context.

Definition 2 The $\Pi_{1}^{0}$-formulas are obtained by universal first order quantification of quantifier-free formulas.

Definition 3 Let $X$ and $Y$ be second order variables and let $x$ and $y$ be first order variables. We introduce the following shorthand notations:

1. The expression $x \in(X)_{y}$ stands for $X(y, x)$.

2. The expression $(X \uparrow Y)(x, y)$ stands for $X(x, y) \wedge Y(x)$.

In order to align our text to the writing style adopted in most texts on monadic second order theories, where second order variables are meant to range over collections of first order objects, from here on and after, we will always write $x \in X$ and $\langle x, y\rangle \in Y$ to mean $X(x)$ and $Y(x, y)$ respectively. We thereby also highlight the fact that, by interpreting relations as sets of tuples, arguments in our base system can be simulated in a theory formulated in the monadic second

\footnotetext{
4 The second author has found a way to generalize the result to parameter free systems to some extent, but with other restrictions on $\prec$. This will be in his future work.
} 
order logic, that in addition to the axioms of minimal logic bears the necessary machinery for an appropriate treatment of ordered pairs (of first order objects). Thus our base system can be embedded into all foundational second order theories of sets and classes (e.g. NBG) or of arithmetic (e.g. RCA $A_{0}$ ).

Further, bold letters are used to denote vectors of either type of variables, and we will use "class terms" such as $\{\mathbf{x} \mid \varphi(\mathbf{x})\}$ for formulas $\varphi(\mathbf{x})$ where, as usual, strings of the form $\mathbf{y} \in\{\mathbf{x} \mid \varphi(\mathbf{x})\}$ are interpreted as $\varphi(\mathbf{y})$.

Definition 4 We also introduce the following shorthand notations:

1. $X \subset Y \equiv \forall \mathbf{x}(X(\mathbf{x}) \rightarrow Y(\mathbf{x}))$;

2. $X=Y \equiv X \subset Y \wedge Y \subset X$.

For the set theoretic context, we use similar notations for first order variables.

Definition 5 Let $\prec$ be a binary relation on $A$, where $x \prec y$ stands for $\prec(x, y)$.

We call $(A, \prec)$ transitive if $\operatorname{tr}(A, \prec)$ holds, where

$$
\operatorname{tr}(A, \prec) \equiv \forall x, y, z \in A(x \prec y \wedge y \prec z \rightarrow x \prec z) .
$$

$A$ collection $Y$ is called progressive in $(A, \prec)$ if $\operatorname{prog}(Y,(A, \prec))$ holds, where

$$
\begin{aligned}
\operatorname{prog}(Y,(A, \prec)) & \equiv \forall a \in A\left(A_{\prec a} \subset Y \rightarrow a \in Y\right), \\
A_{\prec a} & =\{x \in A \mid x \prec a\} .
\end{aligned}
$$

We say that $(A, \prec)$ is well-founded if $\operatorname{wf}(A, \prec)$ holds, where

$$
\operatorname{wf}(A, \prec) \equiv \forall Y(\operatorname{prog}(Y,(A, \prec)) \rightarrow A \subset Y) .
$$

Remark 2 Following our previous discussion on "class terms", for some formula $\varphi(x)$, the expression $\operatorname{prog}(\{x \mid \varphi(x)\},(A, \prec))$ abbreviates

$$
\forall a \in A(\forall b \in A(b \prec a \rightarrow \varphi(b)) \rightarrow \varphi(a)) .
$$

Definition 6 For any relation $(A, \prec)$ and any formula $\psi(X, x)$ let

$$
\operatorname{hier}_{\psi}(H,(A, \prec)) \equiv \forall a\left((H)_{a}=\left\{x \mid \psi\left(H \uparrow A_{\prec a}, x\right)\right\}\right) .
$$

If $\psi$ does not contain the variable $H$ (but possibly other free variables), let

$$
\begin{aligned}
& \psi-\operatorname{TR}(A, \prec) \equiv \exists H \operatorname{hier}_{\psi}(H,(A, \prec)) ; \\
& \psi-\operatorname{TR} \equiv(\forall A, \prec)(\operatorname{wf}(A, \prec) \rightarrow \psi-\operatorname{TR}(A, \prec)) .
\end{aligned}
$$

The expression $\operatorname{hier}_{\psi}(H,(A \prec))$ is often referred to as stating that $H$ is a hierarchy along $(A, \prec)$ of $\psi$ (or of its corresponding operator).

Definition 7 For a class $\mathcal{F}$ of formulas, the scheme $\mathcal{F}$ - $\operatorname{TR}(A, \prec)$ consists of $\psi-\operatorname{TR}(A, \prec)$ and the scheme $\mathcal{F}$-TR consists of $\psi$-TR, both for all $\psi$ from $\mathcal{F}$.

It is customary, when given an effective description $\alpha$ of an ordinal to let $\left(\Pi_{1}^{0}-\mathrm{CA}_{0}\right)_{\alpha}$ and $\left(\Pi_{1}^{0}-\mathrm{CA}\right)_{\alpha}$ denote the systems obtained from adding $\Pi_{1}^{0}-\mathrm{TR}(\alpha)$ to the base theories as given from the context. In arithmetic, the absence of the subscript 0 indicates that induction for arbitrary formulas along both $\alpha$ and $\omega$ is available. See also [5, Subsection 2.4], [2, p. 64] and [6]. Note however, that the theory presented in [6] is parameter free. Also note that the references use slightly different notations; $\Pi_{\alpha}^{0}-\mathrm{CA}_{0}$ in $[5]$ and $\mathrm{ACA}_{\alpha}^{-}$in $[6, \S 4]$. 


\section{Result}

Theorem 1 There is a $\Pi_{1}^{0}$-formula $\varphi(X, Y)$ such that, it is provable within our base system, that if $(A, \prec)$ is transitive, then I below implies II below:

(I) $\exists H \forall a\left((H)_{a}=\left\{y \mid \varphi\left(H \uparrow A_{\prec a}, Y\right)\right\}\right)$;

(II) $\operatorname{prog}(Y,(A, \prec)) \rightarrow A \subset Y$.

Remark 3 The formula $\varphi(X, Y)$ has no free variables other than $X$ and $Y$ and particularly, the variable $y$ does not occur in it. Thus if $H$ is a class as indicated in $(I)$ above, then, classically, $(H)_{a}$ is always either the empty class or the universal class (but we could not say this in intuitionistic setting).

Proof Let $\varphi(X, Y)$ be the $\Pi_{1}^{0}$-formula

$$
\forall a\left(0 \in(X)_{a} \rightarrow a \in Y\right),
$$

where the symbol 0 stands for an arbitrary constant (in the absence of constants, also a free variable can be used instead).

Let $H$ be as stated in $(I)$ above and let $\operatorname{prog}(Y,(A, \prec))$. We have

$$
\forall x, y\left(x \prec y \rightarrow(H)_{y} \subset(H)_{x}\right)
$$

by the transitivity of $\prec$ and the following equivalence for any $x$ and $z$ :

$$
\begin{aligned}
z \in(H)_{x} & \Leftrightarrow \varphi\left(H \uparrow A_{\prec x}, Y\right) \\
& \Leftrightarrow \forall a\left(0 \in\left(H \uparrow A_{\prec x}\right)_{a} \rightarrow a \in Y\right) \\
& \Leftrightarrow \forall a \prec x\left(0 \in(H)_{a} \rightarrow a \in Y\right) .
\end{aligned}
$$

Now we prove

$$
\forall x\left(0 \in(H)_{x} \rightarrow x \in Y\right)
$$

To see this, we use $(2)$ with $z=0,(1)$ and $\operatorname{prog}(Y,(A, \prec))$ to conclude

$$
\begin{aligned}
0 \in(H)_{x} & \Leftrightarrow 0 \in(H)_{x} \wedge \forall a \prec x\left(0 \in(H)_{a} \rightarrow a \in Y\right) \\
& \Rightarrow 0 \in(H)_{x} \wedge \forall a \prec x\left(0 \in(H)_{x} \rightarrow a \in Y\right) \\
& \Rightarrow A_{\prec x} \subset Y \Rightarrow x \in Y .
\end{aligned}
$$

Hence, in view of (3), to see $A \subset Y$ it suffices to show $\forall x \in A\left(0 \in(H)_{x}\right)$. However, this is immediate from (3) and (2) with $z=0$.

Remark 4 Let $\forall(I)$ and $\forall(I I)$ be obtained by universal quantification of $Y$ in $(I)$ and $(I I)$ from the theorem respectively. For any collection $\mathcal{F}$ of formulas that contains the formula $\varphi(X, Y)$ as presented in the proof, the implication $\forall(I I) \rightarrow(I)$ is an instance of $\mathcal{F}-$ TR. Thus $\forall(I)$ and $\forall(I I)$ are equivalent under $\mathcal{F}$-TR and $\operatorname{tr}(A, \prec)$.

Corollary 1 Let $\mathcal{F}$ be a collection of formulas that contains $\Pi_{1}^{0}$ (with parameters). If $(A, \prec)$ is transitive, the schema $\mathcal{F}-\operatorname{TR}(A, \prec)$ entails $\operatorname{wf}(A, \prec)$.

Remark 5 The proof is so straightforward, with the only twist being the duplication (and keeping for several steps) of $0 \in(H)_{x}$ in (4). Thus, pure second order minimal logic is sufficient but the contraction rule is essential. 


\section{Discussions}

\subsection{Consequences}

1. Some theories in the literature (e.g. the theory $\Pi_{\beta}^{0}-\mathrm{CA}_{0}$ in [5]) are formulated in terms of the schema

$$
\operatorname{wf}(A, \prec) \wedge \mathcal{F}-\operatorname{TR}(A, \prec) \wedge \ldots
$$

In these theories, as long as the formula $\varphi(X, Y)$ from the theorem is allowed to instantiate $\mathcal{F}$, the clause $\operatorname{wf}(A, \prec)$ can be dropped. Nevertheless, $\left(\Pi_{1}^{0}-\mathrm{CA}\right)_{\alpha}$ from [2] contains transfinite induction for arbitrary formulas and, at this stage, it is not clear whether transfinite induction for arbitrary formulas can be dropped. This will be clarified in the second author's future work.

2. Theories that suppose hierarchies along ill-founded ${ }^{5}$ relations for a class of formulas containing $\varphi(X, Y)$ are inconsistent. That is, for some formula $\Phi(X)$ if $\exists X(\Phi(X) \wedge \neg \mathrm{wf}(X))$ is provable then any theory containing

$$
\Phi(\prec) \rightarrow \exists H \operatorname{hier}_{\varphi}(H,(A, \prec))
$$

is inconsistent.

4.2 Pseudohierarchies and the $\Pi_{1}^{1}$ completeness of well-foundedness

Under the transfinite recursion $\mathcal{F}$-TR for $\mathcal{F}$ of which the formula $\varphi$, as displayed in the proof of Theorem 1 , is a legit instance, our result seemingly implies

$$
\operatorname{wf}(A, \prec) \longleftrightarrow \exists H \operatorname{hier}_{\varphi}(H,(A, \prec))
$$

and thus seems in contradiction with the $\Pi_{1}^{1}$ completeness of wf. However, as $\varphi$ has two free variables the schema together with our result merely implies

$$
\operatorname{wf}(A, \prec) \longleftrightarrow \forall Y \exists H \operatorname{hier}_{\varphi}(H,(A, \prec)),
$$

yielding a $\Pi_{2}^{1}$ characterization of wf, which is in line with the logical complexity of wf. Similarly, our theorem does not contradict the existence of pseudo hierarchies. By applying the usual diagonalization argument to

$$
\forall Y \forall(A, \prec)\left(\operatorname{wf}(A, \prec) \rightarrow \exists \operatorname{Hhier}_{\varphi}(H,(A, \prec))\right)
$$

and the $\Pi_{1}^{1}$-completeness of the predicate wf, one obtains

$$
\forall Y \exists(A, \prec)\left(\neg \mathrm{wf}(A, \prec) \wedge \exists H \operatorname{hier}_{\varphi}(H,(A, \prec))\right)
$$

which is not in contradiction to our theorem since $\prec$ depends on $Y$. While the above discussion reveals that our corollary does not contradict established knowledge, it also shows that the parameter $Y$ in $\varphi(X, Y)$ cannot be omitted.

5 The phrase "ill-founded" stands for "not well-founded". 
4.3 The case of non-transitive relations

In our theorem, the only precondition is the transitivity of $\prec$. We do not know whether this is necessary in the current setting. If we drop transitivity, and fix the relation $\prec$ on natural numbers to be defined from $n \prec m \leftrightarrow n=m+1$, then the formula $\varphi(X, Y)$ as presented in the proof does not work anymore. However, if we assume classical logic, basic axioms for $\omega$ and that any illfounded relation $(A, \prec)$ posses infinite descending $\prec$-chains ${ }^{6}$, which amounts to incorporate some form of dependent choice to our base system, then we can safely drop the precondition. A proof can be outlined as follows: Fix $\psi(X, f, x)$ such that any $H$ with $(H)_{a}=\left\{x \mid \psi\left(H \uparrow A_{\prec a}, f, x\right)\right\}$ for all $a \in A$ fulfills

$$
(H)_{f(n)}= \begin{cases}(H)_{f(n+1)} \backslash\left\{\min \left((H)_{f(n+1)}\right)\right\} & \text { if }(H)_{f(n+1)} \neq \varnothing \\ \omega & \text { otherwise. }\end{cases}
$$

Let $f: \mathbb{N} \rightarrow A$ be a descending $\prec$-chain. If $(H)_{f(n)}=\varnothing$ for some $n \in \omega$, then $(H)_{f(n+k)}$ is finite for all $k \in \omega$. Thus $\forall k>0\left((H)_{f(n+k)} \neq \varnothing\right)$ and so $a_{k}=\min \left((H)_{f(n+k+1)}\right)$ form an infinite descending chain in $\omega$. Otherwise, $\forall n(H)_{f(n)} \neq \varnothing$ and so $a_{k}=\min \left((H)_{f(k)}\right)$ is an infinite descending chain in $\omega$. Note that the existence of $\min \left((H)_{f(k)}\right)$ requires classical logic.

4.4 Reducing the order of $Y$ in $\varphi(X, Y)$

Remark 6 Assume we work in a second order set theory on classical logic that contains both class-comprehension for quantifier free formulas and the reflection principle defined below ${ }^{7}$ :

$$
\varphi(\mathbf{x}) \rightarrow \exists u\left(\mathbf{x} \in u \wedge \varphi^{u}(\mathbf{x})\right)
$$

for any elementary formula $\varphi$, where $\varphi^{u}(\mathbf{x})$ stands for the relativization of $\varphi$ to $u$. Then we can express $\operatorname{wf}(A, \prec)$ also by the following elementary formula:

$$
\mathrm{wf}^{\prime}(A, \prec) \equiv \forall x(\operatorname{prog}(\{y \mid y \notin x\},(A, \prec)) \rightarrow A \subset\{y \mid y \notin x\}) .
$$

Proof We assume $\operatorname{wf}^{\prime}(A, \prec), \operatorname{prog}(X,(A, \prec))$ and $a_{0} \in A \backslash X$. Note that

$$
\operatorname{prog}(X,(A, \prec)) \longleftrightarrow \forall a \in(A \backslash X) \exists b \in(A \backslash X)(b \prec a)
$$

by contrapositive. Applying reflection, we get a set $u$ such that $a_{0} \in u$ and

$$
\forall a \in(u \backslash X) \exists b \in(u \backslash X)(b \prec a) .
$$

Thus if we stipulate $y$ for $u \backslash X$, we get $\operatorname{prog}(\{x \mid x \notin y\},(A, \prec))$ and $A \not \subset\{x \mid$ $x \notin y\}$ because $a_{0} \in u$. We have shown the contrapositive of $\operatorname{wf}(A, \prec)$.

The reversal follows from a modest comprehension $(\exists X)(X=\{y \mid y \notin x\})$.

\footnotetext{
${ }^{6}$ A function $f: \mathbb{N} \rightarrow A$ such that $f(n+1) \prec f(n)$ holds for all $n \in \mathbb{N}$.

7 The standard settings such as NBG or ZFC satisfy this. See e.g. [3, Theorem I.12.14].
} 
The preceding remark unveils the following difference when interpreting our base system in arithmetical or set theoretic setting respectively. In the former, as observed earlier, we need the second order free variable $Y$ in the formula $\varphi(X, Y)$ while in the latter it could be replaced by a first order variable.

\subsection{Connection to Steel's theorem [9]}

Montalbán pointed out a close connection to Steel's result from [9], which states that there is no sequence $\left\langle A_{n} \mid n \in \omega\right\rangle$ of subsets of $\omega$ such that

(a) for a fixed arithmetical formula $\theta, A_{n+1}$ is unique $B$ with $\theta\left(A_{n}, B\right)$, and

(b) $\left(A_{n+1}\right)^{\prime} \leq_{T} A_{n}$, i.e. the Turing jump of $A_{n+1}$ is Turing reducible to $A_{n}$.

Transfinite recursion applied to a universal $\Pi_{1}^{0}$ formula along a relation $\prec$ provides a strictly monotone map from $\prec$ to $(-)^{\prime} \leq_{T}(-)$, i.e. $a \prec b$ implies $(H)_{a}^{\prime} \leq_{T}(H)_{b}$. Thus, if $f$ is a descending chain in $\prec$, then $\left\langle(H)_{f(n)}: n \in \omega\right\rangle$ satisfies the conditions (a) and (b) above, but with the arithmetical formula containing $f$ as a parameter. Therefore, it follows from the result of Steel's that $f$ is not arithmetical. Furthermore this shows that, in the case of classical arithmetic, our result follows from the relativized version of Steel's theorem.

In one sense, Steel's theorem is more general than ours: $\theta(A, B)$ is not necessarily $B=\{x \mid \psi(x, A)\}$ but any formula which determines $B$ uniquely from $A$. In another sense, however, ours is more general: our base theory is pure minimal logic and hence our result can be applied to intuitionitic theories and also to general second order frameworks (even those without universal formulas or enumerations), whereas the proof that Steel gave is a diagonalization argument and hence relies on the enumeration of $\Sigma_{1}^{0}$ sets.

Acknowledgements The authors would like to express their gratitude to Antonio Montalbán for his valuable comments on an earlier version of the paper, which resulted in Subsection 4.5. Further thanks go to all the members of the Logic and Theory Group of the University of Bern for providing an inspiring work environment.

\section{References}

1. Feferman, S.: Systems of predicative analysis. J. Symb. Log. 29, 1-30 (1964)

2. Feferman, S., Jäger, G.: Choice principles, the bar rule and autonomously iterated comprehension schemes in analysis. J. Symb. Log. 48, 63-70 (1983)

3. Jech, T.: Set Theory, The Third Millennium Edition. Springer (2003)

4. Logic and Theory Group (LTG), IAM, Universität Bern: Subsystems of second order arithmetic and set theory. http://www.ltg.unibe.ch/research/Subsystems $\% 20$ of $\%$ 20Second\%200rder\%20Arithmetic\%20and\%20Set\%20Theory (Accessed 28 May 2014)

5. Marcone, A., Montalbán, A.: Veblen functions for computability theorists. J. Symb. Log. 76, 575-602 (2011)

6. Rüede, C., Strahm, T.: Intuitionistic fixed point theories for strictly positive operators. Math. Log. Q. 48, 159-202 (2002)

7. Sato, K.: Relative predicativity and dependent recursion in second-order set theory and higher-order theories. J. Symb. Log. (2014). Accepted for publication, http://www.iam. unibe.ch/ltgpub/2012/sat12.pdf 
8. Simpson, S.G.: Subsystems of Second Order Arithmetic. Perspectives in Logic. Cambridge University Press (1998)

9. Steel, J.: Descending sequences of degrees. J. Symb. Log. 40, 59-61 (1975) 